Dieses Dokument ist eine Zweitveröffentlichung (Verlagsversion) / This is a self-archiving document (published version):

Alexander Lasch

Texte im Handlungsbereich der Religion

Erstveröffentlichung in / First published in:

Stephan Habscheid, Hrsg. Textsorten, Handlungsmuster, Oberflächen. Berlin/New York:

De Gruyter 2011, S. 536 - 558. ISBN 978-3-11-022930-1.

DOI: https://doi.org/10.1515/9783110229301.536

Diese Version ist verfügbar / This version is available on:

https://nbn-resolving.org/urn:nbn:de:bsz:14-qucosa2-748409 


\title{
Texte im Handlungsbereich der Religion
}

\author{
Alexander Lasch (Kiel)
}

1. Problemaufriss

2. Wissenschaftshistorische Hintergründe und Forschungsstand

3. Kommunikationstypologie religiöser Texte

3.1 (Prophetie und) Verkündigung

3.2 Verehrung

3.3 Vergegenwärtigung: Raum und Zeit, Ritual und Symbol

4. Fazit

5. Literaturverzeichnis

\section{Problemaufriss}

Eine Typologie für Texte, die der Domäne „Religion“ zugeordnet werden sollen, steht vor verschiedenen Problemen, die sich für Texte aus ,profanen' oder ,säkularen' Diskursbereichen nicht stellen. Zum einen ist die Abgrenzung zum ,Profanen` oder ,Säkularen“ und damit die Klärung der Frage, weshalb sprachliche Einheiten als ,religiös' klassifiziert werden, strittig. Zum anderen ist die kommunikative Prägung der Domäne „Religion“ alles andere als knapp zu charakterisieren - dies hängt im Wesentlichen mit der Frage danach zusammen, wer einen Text und dessen Kommunikation (oder/und dessen Vollzug) zu welcher Zeit und an welchem Ort verantwortet. Die letzte Frage betrifft die kommunikativen Besonderheiten der Situationen, in denen die Texte, die der Domäne „Religion“ zugeschrieben werden, kommuniziert werden.

Da eine Kommunikationstypologie für die Domäne „Religion“ bisher fehlt, versucht dieser Artikel kommunikative Grundkonstellationen zu skizzieren, die für die linguistische Beschreibung unerlässlich sind. Es wird hier also nicht darum gehen, Textsortentraditionen verschiedener Glaubensgemeinschaften nach der Art und Weise ihrer Überlieferung oder gar ihrem Status innerhalb der Glaubensgemeinschaften $\mathrm{zu}$ beleuchten, sondern es wird danach gefragt, was die grundlegenden kommunikativen Konstellationen der Domäne „Religion“ sind und unter welchen Bedingungen Texte in der Domäne „Religion“ kommuniziert werden können. 


\section{Wissenschaftshistorische Hintergründe und Forschungsstand}

Die Linguistik scheute sich lange davor, Texte, die der Domäne „Religion“" zugeordnet werden, zu untersuchen. Dem lag sowohl auf Seiten der Linguistik als auch der Theologie das Missverständnis und die Befürchtung zu Grunde, dass man die Deutungskompetenz und Deutungshoheit der jeweils anderen textauslegenden Disziplin damit in Frage stellte. Indiz dafür ist, dass sowohl Hugo Mosers Arbeit zu Sprache und Religion (1964) als auch August Langens Wortschatz des deutschen Pietismus $\left(1968^{2}\right)$ zwar heute noch mit Gewinn rezipiert werden, allerdings in den Folgejahren kaum Arbeiten an ihre Fragestellungen anschlossen. Dass Linguistik und Theologie verschiedene Erkenntnisinteressen haben und unterschiedliche Ziele verfolgen, erwies sich zwar bereits bei Moser und Langen, allerdings öffnete erst die so genannte pragmatische Wende innerhalb der Linguistik, die die Aufmerksamkeit und das Forschungsinteresse weg vom Wort und Satz hin zum Sprachgebrauch lenkte, den Weg zur wissenschaftlichen Begründung des genuin differenten Forschungsinteresses der Linguistik. Jetzt gerieten Fragen der Produktion, Rezeption und Kommunikation in den Blick, die hinsichtlich ihrer Bedingungen und Konsequenzen befragt werden konnten. ${ }^{1}$ Eine weitere Voraussetzung stellte innerhalb der Linguistik die Rezeption der soziologischen Studien zum Ritual dar. Für die Linguistik erwiesen sich dabei sowohl der Gegenstand als auch die Perspektive der Soziologie als wegweisend: Diese abstrahierte nämlich von einzelkulturellen Erscheinungen und suchte generelle Handlungsmuster des Rituellen zu erarbeiten, die sie nicht mehr nur auf die Domäne „Religion“ beschränkt sah. Als einschlägig haben hier die Publikationen von Viktor Turner und Hans Georg Soeffner zu gelten. ${ }^{2}$

Der erste, der aus Perspektive der Linguistik mit dem Interesse der Beschreibung von Kommunikationsstrukturen auf Rituale auch in religiösen Kontexten blickt, ist Iwar Werlen. Seine Monographie Sprache und Ritual (1984), die durch mehrere kleine Beiträge (z. B. Werlen 1987) flankiert wird, wird 1990 ergänzt durch Ingwer Pauls Rituelle Kommunikation, die neben den rituellen kommunikativen Mustern in gottesdienstlichen Feiern auch diejenigen Elemente beschrieb, die individuell verantwortet werden müssen (nämlich im so genannten ,pastoralen Diskurs"). Diese Studie ist auch heute noch als Wegweiser für die Auseinandersetzung mit Kommunikation in religiösen Kontexten heran-

Vgl. exemplarisch Samarin (1983) oder Steger (1984).

2 Vgl. Turner (1989, 1967/1998) und Soeffner (1989). 
zuziehen, ${ }^{3}$ auch wenn sie in der Zwischenzeit besonders in Bezug auf die Ausweitung der Überlegungen auf andere Religionsgemeinschaften und im Hinblick auf die ritualtheoretischen Annahmen der Ergänzung aus linguistischer Sicht bedarf. Letztere lieferte beispielsweise Ulla Fix mit ihrem Band Ritualität in der Kommunikation der DDR (1998), die zum einen darauf hinwies, dass man im Vollzug von Ritualen Oberflächen- und Tiefenfunktionen zu unterscheiden habe, und herausstellte, dass auch Linguisten bei der Analyse und Beschreibung ritueller Handlungen nichtsprachliche Zeichenkomplexe (ästhetisch-visuelle Zeichen, choreographische Zeichen, musikalische Zeichen) zu berücksichtigen hätten (Fix 1998:XVII, XI). Doch auch hier gilt: Nur wenige Arbeiten haben die von Werlen, Paul und Fix aufgeworfenen Fragen in exemplarischen Studien weiterverfolgt. ${ }^{4}$

Besser als um eine Kommunikationstypologie der Domäne „Religion" ist es um die linguistische Beschreibung von Merkmalen der der Kommunikation zu Grunde liegenden Texte bestellt. Besonders in diachroner Perspektive sind einzelne Textsorten beginnend mit dem Althochdeutschen Gegenstand sprachwissenschaftlicher Untersuchung geworden. ${ }^{5}$ Daneben gilt ein besonderes Augenmerk seit jeher in den sprachgeschichtlichen Überblicksdarstellungen den Texten der Reformationszeit und besonders Luthers. ${ }^{6}$ Einzeluntersuchungen wurden zwar vorgelegt etwa zu den Textsorten Kirchenlied (Moser 1981; Greule 1992, 1999a und b, 2004), Predigt (Funk 1991; Pfefferkorn 2005a und b; Grözinger 2009; Paul 2009) und Lebensbeschreibung (Lasch 2005) oder zum Themenkomplex Rhetorik und Stilistik der ,religiösen Sprache' (Grözinger 2009; Paul 2009). Diese beleuchten auch den Zusammenhang von Sprache - Religion - Kommunikation, doch sind immer noch mehr Desiderata als Ergebnisse zu formulieren. Typologische Überblicksdarstellungen sind ebenfalls selten. Leider verspricht der Artikel von Franz Simmler (Textsorten des religiösen und kirchlichen Bereichs) im Textlinguistik-Band der Handbücher zur Sprach- und Kommunikationswissenschaft mehr, als er tatsächlich bietet (er beschränkt sich nämlich streng genommen auf die römische Kirche), allerdings ist es dennoch der beste typologische Überblick zum Thema. Dies trifft ebenfalls auf den Beitrag des Theologen Albrecht Grözinger (Rhetorik und Stilistik in der Theologie) im Handbuch Rhetorik und

3 Vgl. dazu die aktualisierte Zusammenfassung aus rhetorisch-stilistischer Perspektive Paul (2009).

4 Vgl. z. B. Pfefferkorn (2005a) und auch Lasch (2005).

5 Vgl. dazu exemplarisch die Arbeiten von Simmler (2000:690), darüber hinaus nach 2000 exemplarisch (2005a u. b, 2007) und Albrecht Greule (Kirchenliedforschung).

6 Neben den sprachgeschichtlichen Überblicken sei hier besonders Besch (2008) erwähnt. 
Stilistik zu, der sich im Wesentlichen auf die Tradierung (biblischer) stilistischer Figuren und Merkmale in Predigt und Liturgie bis hinein in die christliche Lebensführung beschränkt.

\section{Kommunikationstypologie religiöser Texte}

Ziel dieser kurzen Hinführung auf die Merkmale und Bedingungen religiöser Kommunikation ist es, im Sinne einer homogenen Klassifizierung Merkmale herauszuarbeiten, nach welchen sich die kommunizierten Texte der Domäne „Religion“ ordnen lassen. Ich gehe dabei davon aus, dass nicht die Verwendung der Variante eines Symbolsystems („Religiöse Sprache“) das entscheidende Merkmal darstellt, sondern dass sich aus kommunikationstypologischer Perspektive grundsätzlicher zunächst die Konzepte der „Verkündigung“, „Verehrung“ und der „Vergegenwärtigung“ für eine Beschreibung anbieten, die jedoch aus linguistischer Perspektive erarbeitet werden müssen, um dem spezifischen linguistischen (auch wissenssoziologisch motivierten) Interesse gerecht werden zu können. Statt einer Beispielanalyse eines kommunikativen Ereignisses (die Eucharistiefeier erfreut sich in diesem Zusammenhang großer Beliebtheit) werden die folgenden Überlegungen das Christentum (hier genauer die römische und die evangelische Kirche) und den Islam einbeziehen - bisher liegt keine linguistische Studie vor, die aus kommunikationstypologischer Perspektive diese beiden großen Religionen des Abend- und Morgenlandes beschrieben hätte.?

Basal für alle monotheistischen Religionen ist eine Annahme: Es gibt (die) eine Gottheit, die sich der menschlichen Wahrnehmung in der Immanenz grundsätzlich entzieht und aus der Transzendenz aus die Geschicke der Menschen lenkt und steuert - die Trennung zwischen Immanenz und Transzendenz ist dabei eine nachaufklärerische und in unserem Zusammenhang insofern eine künstliche, als damit wesentliche Aspekte religiöser Kommunikation verstellt werden. Deswegen wollen wir diese Scheidung der ,einen Wirklichkeit' in Immanenz und Transzendenz im Folgenden nur als analytische betrachten.

Die Gottheit verantwortet Weltentstehung und die Erschaffung allen Lebens. Sie ist in der Welt, zugleich aber für die Menschen kommunikativ nicht erreichbar - deren kommunikative Möglichkeiten reichen nicht hinter die Grenzen ihrer Wahrnehmung und so ist ihnen der tiefere

7 Die folgenden hinführenden Überlegungen ließen sich auch auf das Judentum übertragen, bei anderen Religionen, vor allem Buddhismus und Hinduismus, bedürften die Relation zwischen Immanenz und Transzendenz und das Konzept von Gottheit notwendiger Präzisierungen. 
Grund der, einen Wirklichkeit' Arkanum. Menschen sind also stets darauf angewiesen, das von ihnen Wahrgenommene vor der Annahme der Existenz einer Gottheit zu interpretieren und in Beziehung zu ihrem eigenen Leben zu setzen. Die Interpretationsmuster und die Interpretamente (bspw. Gnadenerweis vs. Bestrafung) gehen in das gemeinsame Gedächtnis ein, verfestigen sich kollektiv in religiösen Symbolen, bilden Mythen und Glaubenssätze, die immer darauf abzielen, das Leben des Einzelnen in einer Gemeinschaft zu evaluieren und das Arkanum, das Wirken Gottes in der Welt, zu erschließen. Und so ist

,Religiosität' letztlich als Charakteristikum einer sozial institutionalisierten Verhaltens- und Interaktionssorte zu explizieren [...], die bestimmte charakteristische Situationen konstituiert und strukturiert [...]. Eine Äußerung ist religiös, wenn sie ihren Ort in einer Situation hat, die religiös strukturiert ist. ${ }^{8}$

Eine vor diesen Grundannahmen entworfene Kommunikationstypologie wird nicht homogen sein im Sinne einer idealen Typologie, indem sie nur ein Merkmal aufführte, welchem alle Kommunikate beigestellt werden können (Homogenität). Allerdings - und dies ist ein Vorteil der kommunikativen Grundkonstellation zwischen „Verkündigung“ und „Verehrung" als Akten der „Vergegenwärtigung" - sind die zwei kommunikativen Muster im dritten je ohne das andere schlechterdings nicht denkbar: „Verkündigung“ zieht „Verehrung“ nach sich und umgekehrt. Das liegt daran, dass alle kommunikativen Akte, die sich diesen beiden Formen zuordnen lassen, auf einer kommunikativen Achse von - analytisch - der Immanenz zur Transzendenz und umgekehrt liegen und in rituelle Handlungen der „Vergegenwärtigung“ eingebettet sind. ${ }^{9}$ Weiter kann es hier nicht gelingen, alle Elemente der Typologie zuzuordnen (Exhaustivität), die als kommunizierte Texte in den monotheistischen Religionen auszumachen sind. Ich werde mich im Folgenden exemplarisch vor allem auf die zentralen Kommunikationsstrukturen in der römischen und evangelischen Kirche und im Islam konzentrieren. Es wird sich zeigen, dass sich alle kommunizierten Texte und kommunikativen Handlungen - auf einer abstrakten und analytischen Ebene - den beiden zu unterscheidenden kommunikativen Handlungen, der der „Verkündigung“ und der „Verehrung", zuweisen lassen (Monotypie). Die besondere Gebundenheit dieser beiden kommunikativen (Teil-)Handlungen an rituelle Formen, Zeiten und Orte bringt allerdings die Schwierigkeit mit sich, dass im praktischen Vollzug z. B. der Priester sowohl Verkünder als auch als Stellvertreter der Kultgemeinschaft Verehrer Gottes ist. Allerdings - und dies deutete sich bereits in den Grundannahmen an, ist die Zahl der möglichen Typen von kommunizierten Texten und Handlungen (Finitheit) begrenzt.

8 Funk (1991:25) zitiert hier Ingolf U. Dalferth (1981).

9 Zum Verhältnis von vertikaler und horizontaler Kommunikationsrichtung vgl. vor allem Paul (1990:12 u. ö.; 2009) und auch Simmler (2000:682). 


\section{1 (Prophetie und) Verkündigung ${ }^{10}$}

In religiösen Gemeinschaften kommt den Menschen, die näher an der Gottheit zu stehen scheinen als andere, eine besondere Bedeutung zu. Ihnen wurde durch die Gottheit die Gnade zu Teil, dass sie die Grenzen der Wahrnehmung und die kommunikative Grenze zwischen der Gottheit und der Menschheit überschreiten können und so den Willen und das Wort der Gottheit zu den Menschen tragen:

Was den Propheten offenbar geworden ist, und was sie verkünden, das ist eine einheitliche, in sich geschlossene Überzeugung vom Inhalt und Wesen des Lebens, seinen tiefsten Fundamenten und seinen höchsten Zielen. Es ist ein wirklich einheitliches Ganzes, eine geschlossene Überzeugung, die in wenigen Sätzen zusammengefasst werden kann, nicht mehr ein buntes Vielerlei von Gewohnheit, Herkommen, Volkssitten, kultischen und rituellen Forderungen, ekstatischen Äußerungen, moralischen Sätzen. (Bousset 1903:107)

Diese Propheten (von griech. $\pi \rho \circ \varphi \eta \dot{\tau} \eta \varsigma$, der „Ausleger, Wahrsager, Vorhersager, Verkünder") $)^{11}$ - hier bei Bousset aus religionssoziologischer Perspektive mit dem Fokus auf die kulturübergreifende „einheitliche Lebensauffassung“, die den Begriff des „Propheten“" von den biblischen Religionen löst - sind Zeugen der Selbstoffenbarung Gottes. ${ }^{12}$ Sie sind göttlich inspiriert (im Sinne einer momentanen Eingebung Gottes) oder tragen das Charisma, die ,von Gott dauerhaft verliehene Gabe“ (Lang 2001:172), ${ }^{13}$ sind „Sprachrohr“ einer Gottheit. ${ }^{14}$ Sie sind religiös (und bestenfalls materiell) autonom und damit in der Lage, sich über Konventionen und geltende Regeln hinwegzusetzen

10 Bezeichnend ist hier, dass Funk (1991) noch direkt an die theologische Terminologie anschließt und den Begriff der „Verkündigung“ nicht aus linguistischer Perspektive entwickelt, sondern aus der Homiletik übernimmt.

11 Vgl. Klein (1997:473).

12 Vgl. so Lang (2001:170). - Wie Bousset, so Lang, dehnen auch andere Religionssoziologen um 1900 das Konzept des Prophetischen aus. Dies ist wesentlich auch für die Religionssoziologie Max Webers, der u. a. die Auffassung Boussets von der „einheitlichen Lebensauffassung" weiterentwickelt. Vgl. Weber (1976:275). - So auch Klein (1997:475): „Wenn selbst Muhammad als ,der' Prophet nicht mehr unter die Definition des Propheten fällt, weil diese bis in die Details vom alttestamentlichen Prophetenbild geprägt ist, so wird man mit einer solchen Definition der empirischen Wirklichkeit in der Religionsgeschichte nicht mehr gerecht."

13 Hier im Rekurs auf Webers Unterscheidung der „Prophetenrollen“, die - so Lang weiter - auch in der gegenwärtigen Religionssoziologie und Religionsphilosophie nicht weiter ausgearbeitet worden sind (vgl. Lang 2001:172-174). Allerdings sind diese wohl auch bei Weber typologisch nicht streng zu scheiden, sondern lenken den Blick auf unterschiedliche Merkmale desselben Gegenstandes (vgl. Riesebrodt 2001:200). Die Unterscheidung zwischen „exemplarischer" und „ethischer Prophetie“ Webers (vgl. Weber 1976:273) scheint uns aber dennoch bedenkenswert zu sein. Wird im Weiteren von „Propheten“ gesprochen, dann sind auch im Anschluss daran mit Lang (2001:173f.) „ethische Propheten“, „Sendungspropheten“, gemeint, die streng vom Typus der „exemplarischen Propheten“ (z. B. Buddha) zu scheiden sind.

14 Klein (1997:475). 
(Riesebrodt 2001:198). Nicht selten stellen sie das „Priestertum als Hüter der fest gefügten Tradition in Frage“, indem sie als Träger des persönlichen Charismas dem Amtscharisma der Priester entgegentreten (Klein 1997:476). Sie bezeugen die Selbstoffenbarung Gottes vor den Gemeinschaften, in denen die Gottheit im Zentrum der kultischen Verehrung steht. Ihr Erfolg hängt davon $a b$, ob die Gemeinschaft sie als Zeugen der Selbstoffenbarung Gottes anerkennt und ob sie sich gegen die etablierte Priesterschaft (und das öffentliche Gemeinwesen) durchzusetzen vermögen. ${ }^{15}$ Der mit dem Prophetischen konfrontierte Teil der kultischen Gemeinschaft - seine Anhängerschaft -, der hier als Interpretationsgemeinschaft anzusehen ist, kommt zu dem Schluss, dass der Prophet 1) durch die Offenbarung Teil am Wissen der Gottheit hat, die Ursprung allen Wissens und Seins selbst ist, 2) die göttliche Qualität und den Ursprung des Wissens ausstellt und 3) den unverfälschten Transfer dieses Wissens über die ,eine Wirklichkeit' meist mittels des Symbolsystems Sprache garantiert. Der Prophet nimmt also eine Mittlerrolle in der Kommunikation zwischen der kultisch verehrten Gottheit und der Kultgemeinschaft ein, für die er von der Gottheit erwählt wurde. Er wird von der Kultgemeinschaft in diesem speziellen Sinne als Autorität anerkannt.

Die Träger göttlichen Wissens und Charismas sind, wie auch die Mitglieder der Kultgemeinschaft, Menschen. Als solche sind sie an die Verwendung symbolischer Zeichen gebunden, wenn es um den Transfer göttlichen Wissens geht. Wichtigstes symbolisches Zeichensystem ist seit jeher die Sprache, aber keinesfalls das einzige: Bilder, Musik, Artefakte, architektonisch manifest gewordene rituelle Ordnungen können Symbole des Religiösen sein. Ihnen allen ist gemein, dass sie

ihr Noema (das Gemeinte) so [präsentieren], daß sie nicht nur [sc. wie andere Symbole] auf mehr verweisen, mehr bedeuten, als sie darstellen, sondern sie vermögen auch nur in unzureichender Weise das Wesen dessen zu enthüllen, was sie bezeichnen. Sie verschweigen mehr, als sie offenlegen (Dupré 2007:16).

Das Paradoxon, dass die Propheten göttliches Wissen in symbolischer Kommunikation offenbaren, die verwendeten Symbole aber sich und das, worauf sie referieren, nicht zugleich selbst zu enthüllen vermögen, sichert die durch die Kultgemeinschaft zugewiesene Auslegungsmacht des Propheten, zugleich aber verlangt sie auch die Exegese der Symbole und damit des göttlichen Wissens: Da der Gehalt die Symbole ,im Grunde nicht ausgedrückt werden" kann und dieses allen Symbolen gemeine Merkmal hier „dominant“ wird, ist eine besondere Interpreta-

15 Riesebrodt (2001:199). 
tionsleistung schließlich auch von Nöten (Dupré 2007:16). ${ }^{16}$ Die Verwalter dieser Symbole, Priester, folgen den Propheten nach (Lang 2001:176) und beerben diese als Verkünder und Ausleger. Sie sind es, die zum einen rituelles Handeln, kultische Akte und das Wissen um religiöse Symbole und Glaubenssätze organisieren, systematisieren und verwalten. Zum anderen werden sie immer wieder mit dem Prophetischen konfrontiert. Ihre Aufgabe ist es als ,nachprophetische Gestalten" (Lang 2001:175) dann, das prophetische, inspirierte Wort in die Tradition einzubinden und so zu einem Teil von ihr zu machen. Das einmalige Ereignis, das Handeln und Wirken eines ethischen (oder exemplarischen) Propheten, wird so auf Dauer gestellt und tradiert. Allerdings tradieren Priester nicht nur religiöse Inhalte und damit auch Sprache, sondern sie gestalten sie aktiv (mündlich oder schriftlich) mit: „,[N]eben die unmittelbar wirkende Religion tritt [nämlich] die Theologie, welche die Lehre zu erklären, ihre Widersprüche auszugleichen und zu einem einheitlichen System zusammenzufassen sucht.“17 Dieser nach der Differenzierung Webers - theoretischen Tätigkeit ist die praktische Tätigkeit der Priester zur Seite zu stellen, die Verkündigung („Kollektivbelehrung“) und Seelsorge („religiöse Pflege der Individuen") umfasst. ${ }^{18}$

Den inspirierten Propheten und den Priestern, Verwaltern und Interpretatoren religiöser Symbole und den Verkündern des Wortes und Wissens der Gottheit, kommt eine tragende Rolle in religiösen Gemeinschaften zu. Die Handlung der „Verkündigung“ ist dabei wohl das entscheidende Moment, denn hier sprechen sowohl der Prophet als auch der Priester in der Sprache der Menschen von der Gottheit autorisiert das Wort Gottes: Sie bringen Botschaft und vergegenwärtigen damit zugleich die Offenbarung und ihre Sprache. Es ist - um mit Luther zu sprechen - „Auftrag der Kirche, in welchem ihre Freiheit gründet [...], an Christi Statt und also im Dienst seines eigenen Wortes und Werkes durch Wort und Sakrament die Botschaft von der freien Gnade Gottes auszurichten an alles Volk“ (These VI). ${ }^{19}$ Dieses transferierte Wort und Wissen Gottes, mag es auch hinsichtlich seiner symbolischen Qualität

16 Deutlich ist hier noch einmal zu betonen, dass religiöse Symbole dabei nicht eine ,andere Wirklichkeit" sondern die ,eine Wirklichkeit" repräsentieren, alle anderen Symbole sind „profan“. Dass diese ,eine Wirklichkeit“" im nachaufklärerischen Europa vielen „anderen Wirklichkeiten“ gewichen ist, darf nicht darüber hinwegtäuschen, dass sich religiöse Symbolik und damit die Kommunikation in Domänen des „Religiösen“ immer auf diese „eine Wirklichkeit“ bezieht, die „eine Wirklichkeit“, in der das Leben in der Immanenz als durch und von einer tranzendenten Macht Bewirktes erfahren wird.

17 Meyer (1965:I,146); in der Fassung von 1910 auch zitiert bei Lang (2001:176).

18 Weber $(1976: 283)$.

19 Vgl. Dembowski (2002:714). 
wie oben erläutert der Interpretation bedürfen, wird gesammelt, codiert und schließlich ausgelegt. Ungeachtet dessen wird den heiligen Texten des Christentums und Islams die Qualität zugeschrieben, von Gott selbst geoffenbart zu sein.

Kommunikationstypologisch ist die „Verkündigung“ das kommunikative Muster, welches religiöse Kommunikation von anderen maßgeblich unterscheidet. Es ist zu aller erst die „Verkündigung“ und die Interpretation göttlichen Wortes und Wissens, das durch Wort und Handeln zur Anschauung gebracht wird, nicht jedoch primär dessen Sammlung und Archivierung. Das in den heiligen Texten archivierte Wort und Wissen ist Resultat der „Verkündigung“ und wird erst sekundär in der Tradition selbst zur „Verkündigung“.

Die „Verkündigung“ ist als Transferleistung auf einer vertikalen kommunikativen Achse zu bezeichnen, sie ist einseitig und nicht dialogisch organisiert. Das Wissen, welches in besonderer Weise symbolisiert wird, ist prinzipiell nicht durch den Menschen sondern nur durch Gott verfügbar, der sich einem auserwählten Menschen offenbarte. Diesem auserwählten Propheten folgen die Priester einer Religionsgemeinschaft als Verkünder und Stellvertreter nach. Kommunikative Akte (wie Interpretationen von Gottes Wort) lassen sich nur auf einer horizontalen kommunikativen Achse anschließen.

Die Verbreitung des Wortes der einen Gottheit ist das zentrale Anliegen der monotheistischen Religionen. Eine zentrale Rolle nehmen dabei wie gesehen traditionsgeschichtlich die Propheten ein, die Anhänger um sich scharen und schließlich Religionsgemeinschaften stiften, die sich auf die Propheten berufen. Das Christentum mit seinem Propheten Jesus Christus und der Islam mit seinem Propheten Muhammad, die als ethische Propheten wie auch zugleich als exemplarische Propheten zu charakterisieren sind, sind „Religion[en] der umfassenden Synthese, die, ethische und Erlösungsreligion zugleich, durch ihre Ethik weltzugewandt und durch ihren Erlösungsgedanken asketisch [sind]" (Lang 2001:187). Muhammad steht der Tradition nach als Prophet in einer Reihe mit Adam, Abraham, Moses und Jesus Christus. Er verkündet das ihm durch den Erzengel Gabriel offenbarte Wort Gottes, das in seinem Vortrag (Kur'ān, von arabisch kara'a, „lesen, hersagen vortragen $\left.^{\prime \prime}\right)^{20}$ an die - durch die Tradition als verfälscht angesehenen - heiligen Texte des Juden- und Christentums, das AT und NT, anschließt.

Die Propheten bringen Botschaft, sie vermitteln Gotteswort, in ihrem Wort verkündet sich die Gottheit selbst. Deshalb wird sowohl im

20 Vgl. Wensinck (1976:347). 
Christentum als auch im Islam nicht allein dem Propheten, sondern auch dem von ihm vermittelten Wort selbst zugesprochen, Verkünder zu sein. ${ }^{21}$ Weit über ein solches Verständnis geht der Islam hinaus. Der Heilige Text des Kur'ān wurde von Allāh, der dem Propheten durch den Engel das himmlische Buch ins Herz vorlas, in arabischer Sprache geoffenbart und nicht nur sein Inhalt, sondern auch seine Form und seine Sprache gelten als göttlich und damit unantastbar: Da Schrift nicht Wort im Vortrag ist, genießen die Muslime, die den Koran frei rezitieren können, auch heute noch besonderes Ansehen. Eine Übersetzung des Kur'ān in andere Sprachen gilt noch heute in der islamischen Theologie streng genommen als unmöglich, da jede Übersetzung zugleich Interpretation sei.

Christliche Verkündigung ist Verkündigung Jesu Christi (in seiner Einheit mit Gottvater und heiligem Geist), Verkündigung im Islam ist Verkündigung Allāh. Die Priester (der Priester oder Pfarrer der christlichen Kirchen und der Khatib im Islam), ${ }^{22}$ die professionellen nachprophetischen Verkündiger, ziehen dazu die heiligen Texte hinzu. Im Zentrum christlicher Verkündigung steht das NT, daneben spezieller noch die Prophetien im AT, weiter die Archetypen des AT, die in der exegetischen Tradition kontrafaktisch Jesus Christus gegenübergestellt werden und dem Trinitätsgedanken gemäß prinzipiell auch das vollständige AT. ${ }^{23}$ Im Islam steht der Kur'ān mit seinen 114 Suren im Mittelpunkt der Verkündigung, von denen 113 mit einer Huldigungsformel eingeleitet werden (Basmala, ,im Namen Gottes, des barmherzigen Erbarmers" $)^{24}$ und damit zugleich die Nähe zur kommunikativen Formen der „Verehrung" anzeigen.

Lesungen und Rezitationen (der Bibel oder des Kur'ān) und Predigten (Homilie und Khutba) ${ }^{25}$ sind die wesentlichen kommunikativen Formen der „Verkündigung“ in Christentum und Islam, zugleich entziehen sie sich ritueller Überformung, auch wenn ihnen natürlich Textsortenmuster zu Grunde liegen. Die Priester tragen diese im Gottesdienst vor, den die Christen am Sonntag und die Muslime am Freitag in ihrem Gotteshaus, der Kirche und der Moschee, feiern. Die Geschichte dieser Textsorten, die als Formen der „Verkündigung“ aus kommunikationstypologischer Perspektive im Zentrum gottesdienstlichen Geschehens stehen, ist durch die Linguistik noch nicht befriedigend aufgear-

21 Dembowksi (2002).

22 Vgl. Wensinck (1976:310).

23 Zur Predigt vgl. Simmler (2000:683f.) und Paul (1990).

24 Vgl. Wensinck (1976:78).

25 Vgl. Wensinck (1976:321). 
beitet. $^{26}$ Aus kultureller und religionsvergleichender Perspektive sind für Linguisten dabei weniger Lesungen und Rezitationen, sondern vielmehr die Predigten interessant. Diese dienen nicht allein der Verkündigung von Gotteswort (in vertikaler Kommunikationsrichtung), sondern lassen daneben für den Priester (in horizontaler Kommunikationsrichtung) einen breiten Spielraum, ${ }^{27}$ welcher Interpretation er das verkündete Wort Gottes zuführt und in Bezug zur gesellschaftlichen, kulturellen und politischen Gegenwart setzt. Während in der römischen Kirche erst im 19. und 20. Jahrhundert die Wortpredigt über die Sonntagsgottesdienste hinaus an Gewicht gewinnt, ${ }^{28}$ bildet in der evangelischen Kirche die Wortpredigt das Zentrum des Gottesdienstes. In ihr wird nicht nur das Wort Gottes verkündet, sondern darüber hinaus auf aktuelle gesellschaftliche, politische und kulturelle Ereignisse Bezug genommen. Bereits in der Mitte des 16. Jahrhunderts trägt sie damit ganz wesentlich zur Schärfung des konfessionellen Profils der evangelischen Kirche bei. ${ }^{29}$ Ähnliches gilt auch für den Gottesdienst im Islam: Neben den obligatorischen Bestandteilen der Khutba, den Gebeten über den Propheten und für die Gläubigen und Rezitationen von Ausschnitten aus dem Kur'ān, dienen die Predigten des Khatib (bis zur Trennung der religiösen von der politischen Domäne) auch dazu, auf politische Entwicklungen Bezug zu nehmen und politische Entscheidungen $\mathrm{zu}$ verkünden. Die Khutba geht im Freitagsgottesdienst dem rituellen Gebet (Salāt) voraus. ${ }^{30}$

Neben Lesungen/Rezitationen und Predigten sind auch alle Formen ritueller Kommunikation als verkündigend einzustufen, die das Wort und den Namen Jesu Christi oder Allāhs im Ritual vergegenwärtigen. Dazu zählen die christlichen Formeln des Friedensgrußes oder die Einsetzungsworte in der Eucharistiefeier, mit denen der Priester direkt die in den Evangelien überlieferten Worte Jesu Christi wiedergibt. Anrufungen und Huldigungen der Gottheit (Anrufung der Trintität und die Basmala) bilden kommunikationstypologisch die Brücke zu den Formen der „Verehrung“, sofern sie durch den professionellen Vertreter der Religionsgemeinschaft vorgetragen werden. Das Dilemma, dass hier vom Sprecher her die Texte einem kommunikativen Ereignis zuge-

26 Die Forderung von Simmler (2000:685) nach ausführlichen Text(sorten)analysen ist deswegen hier erneut zu wiederholen.

27 Vgl. Paul (1990:12).

28 Vgl. Simmler (2000:684).

29 Vgl. Funk (1991) - Weiter konnte dies am Beispiel der Hochzeitspredigt aus dem Blick der Geschichtswissenschaften jüngst Markgraf (2007) zeigen.

30 Vgl. Wensinck (1976:321f.) - Der Salāt ist die ,im Arabischen übliche Bezeichnung des rituellen Gebetes oder Gottesdienstes" und kann nicht ohne weiteres einfach als „Gebet“ wiedergeben werden. Vgl. Wensinck (1976:636). 
ordnet werden müssen, lässt sich auch kommunikationstypologisch nicht auflösen.

\subsection{Verehrung}

Der „Gottesdienst“, die Gottesverehrung innerhalb einer religiösen Gemeinschaft, einer Kultgemeinschaft, bildet den zweiten zentralen Komplex kommunikativer Handlungen: Der Begriff wird religionswissenschaftlich verwendet für ,eine heilige Handlung, die in rituell feststehenden Formen von einer Gemeinschaft vollzogen wird, in deren Vertretung ein oder mehrere Zelebranten die gottesdienstlichen Akte vollziehen. ${ }^{\text {(31 }}$ Krux ist und bleibt, dass die verehrte Gottheit nicht auf ein Kommunikationsangebot einer Kult- oder Glaubensgemeinschaft, die durch das gleiche Bekenntnis verbunden ist, direkt antwortet. Meist sind es nur die Zeichen in der, einen Wirklichkeit', die zur Interpretation des Gotteswillens zur Verfügung stehen - und hier kommen die Priester zu ihrem Recht. Sie verwalten die systematisch geordnete Tradition und führen als Stellvertreter einer Glaubensgemeinschaft die zentralen rituellen Handlungen durch und realisieren die kommunikativen Muster, die sich als erfolgreich erwiesen haben. Grundlegend ist also die Annahme der ,einen Wirklichkeit', in der der rituelle Vollzug reale Wirkungen verursacht. ${ }^{32}$ Daneben jedoch bietet die Interpretation der , einen Wirklichkeit' noch weitere Wege, die dem Propheten nicht zur Verfügung stehen. Während dem Propheten bei Nichtakzeptanz bestenfalls die Anhänger kündigen, verfügt der Priester mit der systematisch geordneten Tradition über fein abgestimmte Register, um die Schuld für scheiternde Kommunikation und den daraus erwachsenden negativen Konsequenzen von sich auf die verehrte Gottheit oder das Fehlverhalten der Kult- oder Glaubensgemeinschaft zu lenken. ${ }^{33}$

Die zentralen kommunikativen Formen der ,Verehrung“ sind das Bekenntnis (das Credo und der Tashahhud) ${ }^{34}$ und das Gebet (Vaterunser und Salāt). ${ }^{35}$

Unter einem Glaubensbekenntnis versteht man eine formelhafte, in ihrem Wortlaut festliegende und meistens, jedoch nicht immer, sehr knappe Zusammenfassung der Kernpunkte einer Religion. (Lanczkowski 1993a:384)

31 Lanczkowksi (1993b:1).

32 Lanczkowksi (1993b:1).

33 Weber (1976:261).

34 Vgl. Wensinck (1976:638-640).

35 Sämtliche anderen Formen der Verehrung (wie musikalische Gestaltung, Gesang von Kirchenliedern durch die Gemeinde oder einen Chor etc.) können hier nicht im Detail beschrieben werden - sie unterscheiden sich für die je zu charakterisierende Glaubensgemeinschaft auch zu deutlich voneinander. 
In der Ostkirche wird das Nizänum gebetet, in den christlichen Kirchen des Westens das Apostolikum. ${ }^{36}$ Es dient - hier in der ökumenischen Fassung - dem ,gottesdienstliche[n] Lobpreis“ und ist „verbindliche Lehraussage“ $:^{37}$

Ich glaube an Gott, den Vater, den Allmächtigen, den Schöpfer des Himmels und der Erde. | Und an Jesus Christus, seinen eingeborenen Sohn, unsern Herrn, empfangen durch den Heiligen Geist, geboren von der Jungfrau Maria, gelitten unter Pontius Pilatus, gekreuzigt, gestorben und begraben, hinabgestiegen in das Reich des Todes, am dritten Tage auferstanden von den Toten, aufgefahren in den Himmel; er sitzt zur Rechten Gottes, des allmächtigen Vaters; von dort wird er kommen, zu richten die Lebenden und die Toten. | Ich glaube an den Heiligen Geist, die heilige christliche Kirche, Gemeinschaft der Heiligen, Vergebung der Sünden, Auferstehung der Toten und das ewige Leben. Amen

Im Islam ist das Glaubensbekenntnis (Tashahhud) der erste von „fünf Grundpfeilern des Islams“ - es wird bei der Salāt, dem rituellen Gebet, von jedem Gläubigen täglich fünfmal in sitzender Position und im Freitagsgottesdienst - hier ist der Imām ritueller Vorbeter $^{38}$ - nach dem Adhān (,Ankündigung“) $)^{39}$ des Mu'adhhin ${ }^{40}$ vom Minarett der Moschee vollzogen. Der Adhān selbst ist formelhaft und besteht (bei orthodoxen Muslimen) aus sieben Formeln: „Gott ist der Größte“ | „Ich bezeuge, dass es keinen Gott gibt außer Allāh" | „Ich bezeuge, dass Muhammad Gottes Gesandter ist“| „Auf zur salāt!“| „Auf zum Heil!“| „Gott ist der Größte" | Es gibt keinen Gott außer Allāh". ${ }^{41}$ Das Glaubensbekenntnis folgt dem Rezitieren von rituellen Einleitungsformeln, die der Muslim für sich oder gemeinsam mit dem Imām (beim Freitagsgottesdienst) spricht: ${ }^{42}$ „Ich bezeuge, dass es keine Gottheit gibt außer Gott; ich bezeuge, dass Muhammad der Gesandte Gottes ist." 43

Beide Bekenntnisse sind in Religionsgemeinschaften kommunikationstypologisch der kommunikative Akt, der die "Rechtgläubigkeit" des Einzelnen vor sich und anderen Mitgliedern der Gemeinschaft und schließlich vor Gott und den Verkündigern seines Wortes ausweist. ${ }^{44}$ Der Vollzug dieser kommunikativen Handlung ist Bedingung, an der

36 Barth (1993).

37 Im Wortlaut und als PDF-Dokument auf der Internetpräsenz der Evangelischen Kirche in Württemberg:

$<$ http://www.elk-wue.de/glauben/glaubenstexte/bekenntnisse/apostolischesglaubensbekenntnis/> (Stand: 01.12.2010).

38 Vgl. Wensinck (1976:206-208).

39 Vgl. Wensinck (1976:17).

40 Vgl. Wensinck (1976:17, 517).

41 Wensinck (1976:17).

42 Vgl. Wensinck (1976:638f.).

43 Tworuschka (2002:181) und Lanczkowski (1993a:385).

44 Vgl. Wengst (1984:398). 
Gemeinschaft überhaupt teilzuhaben (im Islam wird man durch das öffentliche Sprechen des Bekenntnisses vor Zeugen Muslim).

Während im Islam Gebet und Glaubensbekenntnis in der Salāt untrennbar miteinander verbunden sind, nimmt im Christentum das Vaterunser als Gebet des Herrn eine herausragende Stellung gegenüber dem Glaubensbekenntnis und anderen Gebeten ein. Gebete sind die kommunikativen Handlungen, in denen sich der Gläubige (in seiner Gemeinschaft) der verehrten Gottheit zuwendet, sie direkt anspricht die Bedeutung des Gebets lässt sich in einer Sentenz religionsgeschichtlicher Forschung so zusammenfassen: Ohne Gebet keine Religion. ${ }^{45}$ Das Vaterunser der christlichen Kirchen, das Gebet des Herrn, welches er nach dem NT seine Jünger lehrte (Lk 11,2-4, Mt 6,9b-13 und Did 8,2f.), gilt in der heutigen Fassung nach Nestle/Aland als zuverlässig. ${ }^{46}$ Es hat einen festen Platz in der gottesdienstlichen Verehrung ${ }^{47}$ und zeichnet sich vor allem dadurch aus, dass es nicht - wie das Glaubensbekenntnis - Interpretationen ausschließt, sondern gerade in seiner „Offenheit" sich einer „durchgehende[n] eschatologische[n] Interpretation“ entzieht und damit inkludierend wirkt. ${ }^{48}$

\subsection{Vergegenwärtigung: Raum und Zeit, Ritual und Symbol ${ }^{49}$}

Neben der Sprache, ${ }^{50}$ die alle genannten Formen des gottesdienstlichen Vollzugs transportiert und tradiert, sind Gesang und Fürbitten Bestandteil der „Verkündigung“ und „Verehrung“. Rituelle Handlungen können darüber hinaus durch Musik (instrumental/Gesang), Tanz, Festzüge und Prozessionen begleitet werden. ${ }^{51}$ „Verkündigung“ und „Verehrung" sind deswegen zwar prinzipiell jederzeit und an jedem Ort möglich, religionsgeschichtlich ist aber zu konstatieren, dass der Gottesdienst eine "konservative Eigenart" aufweist, ${ }^{52}$ in dem mit den fest gefügten Ordnungen und Handlungen meist auch Zeiten, Räume und Rituale verbunden sind. In „Verkündigung“ und „Verehrung“ wird eben nicht singulär allein das Wort Gottes rezitiert oder die Gottheit einmalig angerufen, sondern alle Akte der „Verkündigung“ und „Verehrung" werden gleichsam vergegenwärtigt im Vollzug ritueller Handlungen an einem heiligen Ort zu einer heiligen Zeit, an einem Heterotopos mit der ihm eigenen Zeitstruktur. Daher zählen neben den

\footnotetext{
45 Vgl. Ratschow (1993:31).

46 Vgl. Luz (2002:504).

47 Vgl. Seitz (2002:524f.).

48 Luz (2002:510).

49 Vgl. dazu ausführlich Sundermeier (1999:68-97).

50 Vgl. dazu im besonderen Dupré (2007:45-68).

51 Lanczkowksi (1993b:4).

52 Lanczkowksi (1993b:2).
} 
kommunikativen Haushalten auch Heterotopien und Heterochronien zu den Rahmenbedingungen für „Verkündigung“ und „Verehrung“. ${ }^{53} \mathrm{He}-$ terotopien sind Gegenräume, Orte, die zwar lokalisierbar sind, aber „reale Orte jenseits aller Orte“ bleiben. Die heiligen Orte, an denen Kultgemeinschaften ihre Gottheit verehren, bringen ,an ein und demselben Ort mehrere Räume zusammen, die eigentlich unvereinbar sind“ (Foucault 2005:14), „stets ein System der Öffnung und Abschließung besitzen, welches sie von der Umgebung isoliert" (Foucault 2005:18) und ,alle anderen Räume in Frage“ (ebd.:19) stellen. Räume dieser Art gehen oft mit Heterochronien zusammen, sie sind Orte einer anderen Zeit (Ewigkeit, Moment, Übergang) (Foucault 2005:16). Die Heterotopien sind die Häuser Gottes, die nach außen den Raum der „Verkündigung“ und „Verehrung“ abschließen, man nennt sie Tempel, Kirche oder Moschee. ${ }^{54}$ Je nach Religionsgemeinschaft sind die Gotteshäuser architektonisch auf eigene Art und Weise gestaltet und nehmen in ihrer symbolischen Ausgestaltung Glaubenspunkte des jeweiligen Bekenntnisses auf (das strenge Bilderverbot des Islams kennt das Christentum beispielsweise nicht). Doch ist das Gotteshaus nicht der einzige Heterotopos, sondern im Islam auch beispielsweise der Platz hinter der Sutra. Es ist der Gebetsplatz des Muslims, den er mit der Sutra fiktiv nach außen abgeschlossen hat. ${ }^{55}$

In diesen Heterotopien spielen rituelle Handlungen, die den Ort zu einem Heterotopos mit einer eigenen Zeitstruktur machen, eine zentrale Rolle. Rituale sind wiederholte Handlungen, ${ }^{56}$ die zu charakterisieren sind als symbolische, institutionalisierte, ${ }^{57}$ stereotypisierte und expres-

53 Zum Begriff der Heterotypie vgl. Foucault (2005). - Foucault entwirft das Konzept der Gegenräume, der Orte, die lokalisierbar sind, aber „reale Orte jenseits aller Orte“ bleiben. Besonders wird in unserem Zusammenhang interessieren, dass „Heteropien an ein und demselben Ort mehrere Räume zusammen[bringen], die eigentlich unvereinbar sind“ (ebd.:14), „stets ein System der Öffnung und Abschließung besitzen, welches sie von der Umgebung isoliert“ (ebd.:18) und „alle anderen Räume in Frage“ stellen (ebd.:19). Heterochronien - Orte einer anderen Zeit (Ewigkeit, Moment, Übergang) gehen oft mit den Heterotopien zusammen (vgl. ebd.:16).

54 Auf einen besonderen Heterotopos kann hier nur in einer Anmerkung hingewiesen werden: Der Pilgerort und die Pilgerfahrt. Ein lokalisierbarer Ort in der Welt wird hier zum ideellen Zentrum für die Religionsgemeinschaft erklärt, um den sich die Gläubigen sternförmig angeordnet sehen. Sowohl der Ort als auch der Weg dahin bilden gemeinsam ein Heterotop. Die Pilgerfahrt spielt in der römischen Kirche und im Islam - hier ist die Reise nach Mekka eine der „fünf Säulen“ - eine herausragende Rolle.

55 Vgl. Wensinck (1976:708).

56 Hier zunächst undifferenziert im Hinblick auf die Frage nach dem Muster/vs. der Realisierungen des Musters. Vgl. dazu Elspaß (2001:29) und Lasch (2005:73-75).

57 Damit wird auf die Bedingung der Möglichkeit zur Wiederholung von Handlungen verwiesen. Die Handlungen werden durch eine Institution legitimiert und bestätigen dadurch nicht nur sich selbst, sondern auch die Werte und Normen derselben. 
sive Handlungen oder Handlungsfolgen, die auch immer zugleich noch instrumentell sind. Handeln Teilnehmer einer Kultgemeinschaft im Ritual, dann wird Wissen (dessen sich alle Teilnehmer einig sind) vermittelt, zur Schau gestellt und gewonnen - hier wird rituelle Bedeutung im Vollzug hergestellt. Das Handeln selbst ist als Intentional-NichtIntentionales Handeln zu bestimmen, denn nicht nur die Handlungen selbst sind vorgegeben, sondern auch die Handlungsträger werden bestimmt. Die Körper der Handlungsträger werden im Raum angeordnet und konstituieren somit rituellen Raum - den Heterotopos, der sich in den meisten Religionen dann auch architektonisch verfestigt. Rituelles Handeln ist zeitlich und räumlich unmittelbar, es gestaltet die Gegenwart durch die Vergegenwärtigung des Vergangenen. ${ }^{58}$ Was im Islam der Salāt, ist im Christentum die Feier des Abendmahls. In diesen zentralen Ritualen, die vordergründig wie Predigt nicht nur auf das Symbolsystem Sprache setzen, laufen kommunikationstypologisch sowohl „Verkündigung“ als auch „Verehrung“ in der „Vergegenwärtigung“ zusammen, hier sind in jeder Realisierung des Rituals die Glaubenspunkte der Religionsgemeinschaft präsent.

Die kommunikativen Handlungen, die der „Verkündigung“ und der „Verehrung“ zuzuordnen sind, sind immer an heilige Orte und Zeiten gebunden und in Riten eingebettet. Hier bewegen sich Körper nach einer abgestimmten Choreographie, sie nehmen eine bestimmte Haltung an (zum Beispiel beim Gebet). „Verehrung“ und „Verkündigung“ sind deswegen nicht an die Verwendung von Sprache gebunden, sondern können eher von religiösen Symbolen nicht losgelöst betrachtet oder gar verstanden werden. Das gilt selbst auch für das zentrale Symbolsystem, die Sprache ${ }^{59}$ die gleichsam durch die „Verkündigung“, „Verehrung“ in ritueller „Vergegenwärtigung“ an heiligem Ort sakralisiert wird. Diese Sprache kann Elemente eines älteren Idioms der in einer Gemeinschaft aktuellen Verkehrssprache tragen oder sie kann ein völlig anderes Idiom sein (wie das Latein in der römischen Kirche vor dem 2. Vatikanischen Konzil oder das Arabische im Islam).$^{60}$ Der Vollzug

der gottesdienstlichen Akte ist formal konstituiert durch Worte und Handlungen [...]. Spezifische Formen der durch Tradition geheiligten Worte sind Huldigungen der Gottheit in Hymnen und Psalmen, der Vortrag des Kultmythos bzw. der Kultlegende, Gebete und priesterlicher Segen, eventuell auch ein kultisches Orakel (Lanczkowksi 1993b:3).

58 Vgl. dazu Turner (1989,1967); Soeffner (1989); Werlen (1984); Paul (1990); Fix (1998); Kauke (1998); Belliger \& Krieger (1998); Rappaport (1998).

59 Die Gründe für den „konservierenden Charakter der religiösen Sprache“ sind auch zum guten Teil hierin zu suchen. Zuletzt formulierte diese in Anschluss an Moser (1964:45); Funk (1991:18).

60 Lanczkowksi (1993b:2). 


\section{Fazit}

Eine Beschreibung kommunikationstypologischer Gemeinsamkeiten dreier Religionsgemeinschaften in einem Handbuch muss sich zwangsläufig beschränken. Zahlreiche rituelle Ausformungen und Besonderheiten verschiedener Religionsgemeinschaften - und dies auch in historischer Perspektive - können so nicht angemessen gewürdigt und dargestellt werden.

Eine textlinguistische Beschreibung, die sich vor allem auf die tradierten Archive von Religionsgemeinschaften bezieht, kann die kommunikativen Besonderheiten der Domäne „Religion“ nur unzureichend erfassen. Denn die erarbeiteten Komplexe kommunikativen Handelns, die „Verkündigung“ und die „Verehrung“ in der rituellen „Vergegenwärtigung“", prägen das gesamte Gefüge des kommunikativen Haushaltes der abend- und morgenländischen Religionsgemeinschaften.

Religiöse Kommunikation ist aus linguistischer Perspektive überhaupt nur so zu beschreiben. Dabei ist es zunächst trotz aller Sorgfalt unerlässlich, die Ebenen der „Verkündigung“ und „Verehrung“ in der „Vergegenwärtigung“ in der analytischen Beschreibung zu trennen. Allerdings muss dabei stets bewusst gehalten werden, dass die Kommunikation in der Domäne „Religion“" stets auf die ,eine Wirklichkeit" abzielt. Prophet und Priester - die von außen betrachtet auf der Grenze zwischen Immanenz und Transzendenz zu stehen scheinen - sind der ,einen Wirklichkeit' genauso zugehörig wie die Mitglieder einer Religionsgemeinschaft und die Wirkungen der von ihr angebeteten Gottheit. Zahlreiche Missverständnisse im Dialog der Religionen (und auch in ihrer Erforschung) basieren darauf, dass man diese Besonderheit der Kommunikation in der Domäne „Religion“ in westlichen Gesellschaften scheinbar vergessen hat.

\section{Literaturverzeichnis}

Barth, Hans-Martin: „Apostolisches Glaubensbekenntnis. Reformations- und Neuzeit.“ In: TRE 3 (1993), 554-566.

Belliger, Andréa u. David J. Krieger: „Einführung.“ In: Ritualtheorien. Ein einführendes Handbuch. Hg. v. Andrea Belliger u. David J. Krieger. Opladen, Wiesbaden 1998, 7-33.

Besch, Werner: Deutscher Bibelwortschatz in der frühen Neuzeit. Auswahl-Abwahl-Veralten. Frankfurt/Main 2008.

Bousset, Wilhelm: Das Wesen der Religion, dargestellt an ihrer Geschichte. Halle/Saale 1903.

Dalferth, Ingolf U.: Religiöse Rede von Gott. München 1981.

Dembowski, Hermann: „Verkündigung. Dogmatisch.“ In: TRE 34 (2002), 714-717. 
Dupré, Louis: Symbole des Heiligen. Die Botschaft der Transzendenz in Sprache, Bild und Ritus. Übers. u. hg. v. Heinz Grosch unter Mitarbeit v. Alice Fleming-Corten. Freiburg/Breisgau, Basel, Wien 2007.

Elspaß, Stephan: „Sprachliche Ritualisierung des Gedenkens. Zur Geschichte der öffentlichen Reden zum 20. Juli 1944." In: Sprache und Literatur in Wissenschaft und Unterricht 87 (2001), 27-56.

Fix, Ulla: „Ritualität im Wandel - Projektbericht.“ In: Ritualität in der Kommunikation der DDR. Ergänzt durch eine Bibliographie zur Ritualität. Hg. v. Ulla Fix. Frankfurt/Main 1998, IX-XXII.

Foucault, Michel: Die Heterotopien - Der utopische Körper. Zwei Radiovorträge. Frankfurt/Main 2005.

Funk, Tobias: Sprache der Verkündigung in den Konfessionen. Tendenzen religiöser Sprache und konfessionsspezifischer Varianten in den deutschsprachigen Predigten der Gegenwart. Frankfurt 1991.

Greule, Albrecht: „Über den Beitrag der Sprachwissenschaft zur Kirchenliedforschung. Drei mögliche Zugriffe.“ In: ZfdPh 111 (1992), 65-77.

Greule, Albrecht: „Die Sprache im Neuen geistlichen Lied.“ In: Kirchenlied interdisziplinär. Hg. v. Hermann Kurzke u. Hermann Ühlein. Frankfurt/Main 1999a, 83-98.

Greule, Albrecht: „So sie’s nicht verstehen, so sollten sie's nicht singen? Über den Beitrag der Sprachwissenschaft zur Kirchenliedforschung. “In: Kirchenlied interdisziplinär. Hg. v. Hermann Kurzke u. Hermann Ühlein. Frankfurt/Main 1999b, 47-64.

Greule, Albrecht: „Gesangbuch und Kirchenlied im Textsortenspektrum des Frühneuhochdeutschen." In: Textsortentypologien und Textallianzen von der Mitte des 15. bis zur Mitte des 16. Jahrhunderts. Hg. v. Franz Simmler. Berlin 2004, 521-533.

Grözinger, Albrecht: „Rhetorik und Stilistik in der Theologie.“ In: Rhetorik und Stilistik. Ein internationales Handbuch zeitgenössischer Forschung. Rhetorik und Stilistik Halbbd. 2. Hg. v. Ulla Fix, Andreas Gardt u. Joachim Knape. Berlin, New York 2009, 1798-1810.

Kauke, Wilma: „Ritualbeschreibung am Beispiel der Jugendweihe.“ In: Ritualität in der Kommunikation der DDR. Ergänzt durch eine Bibliographie zur Ritualität. Hg. v. Ulla Fix. Frankfurt/Main 1998, 101-214.

Klein, Wassilois: „Propheten/Prophetie. Religionsgeschichtlich.“ In: TRE 27 (1997), 473-476.

Lanczkowski, Günter: „Glaubensbekenntnis. Religionsgeschichtlich.“ In: TRE 13 (1993a), 384-386.

Lanczkowski, Günter: „Gottesdienst. Religionsgeschichtlich.“ In: TRE 14 (1993b), $1-5$.

Lang, Bernhard: „Prophet, Priester, Virtuose.“ In: Max Webers ,Religionssystematik`. Hg. v. Hans G. Kippenberg u. Martin Riesebrodt. Tübingen 2001, 167-191.

Langen, August: Der Wortschatz des deutschen Pietismus. Tübingen $1968^{2}$.

Lasch, Alexander: Beschreibungen des Lebens in der Zeit. Zur Kommunikation biographischer Texte in den pietistischen Gemeinschaften der Herrnhuter Brüdergemeine und der Dresdner Diakonissenschwesternschaft im 19. Jahrhundert. Münster 2005.

Luz, Ulrich: „Vaterunser. Neues Testament.“ In: TRE 34 (2002), 504-512. 
Margraf, Erik: Die Hochzeitspredigt der Frühen Neuzeit. Mit einer Bibliographie der selbständig erschienenen Hochzeitspredigtdrucke der Herzog-AugustBibliothek Wolfenbüttel, der Staats- und Stadtbibliothek Augsburg und der Universitätsbibliothek Augsburg. München 2007.

Meyer, Eduard: Geschichte des Altertums. 5 Bde. in 8 Teilbde. Bd. 1/1. Darmstadt $1965^{7}$.

Moser, Dietz-Rüdiger: Verkündigung durch Volksgesang. Studien zur Liedpropaganda und-katechese der Gegenreformation. Berlin 1981.

Moser, Hugo: Sprache und Religion. Zur muttersprachlichen Erschließung des religiösen Bereichs. Wirkendes Wort Beiheft 7. Düsseldorf 1964.

Paul, Ingwer: Rituelle Kommunikation. Sprachliche Verfahren zur Konstitution ritueller Bedeutung und zur Organisation des Rituals. Tübingen 1990.

Paul, Ingwer: „Rhetorisch-stilistische Eigenschaften der Sprache von Religion und Kirche." In: Rhetorik und Stilistik. Ein internationales Handbuch zeitgenössischer Forschung. Rhetorik und Stilistik Halbbd. 2. Hg. v. Ulla Fix, Andreas Gardt u. Joachim Knape. Berlin, New York 2009, 2257-2274.

Pfefferkorn, Oliver: „Predigt und Andacht als Textsorten der protestantischen Erbauungsliteratur des 17. Jahrhunderts.“ In: ZfdPh 124 (2005a), 375-394.

Pfefferkorn, Oliver: ,Übung der Gottseligkeit. 'Die Textsorten Predigt, Andacht und Gebet im deutschen Protestantismus des späten 16. und des 17. Jahrhunderts. Deutsche Sprachgeschichte. Texte und Untersuchungen 1. Frankfurt/Main 2005b.

Rappaport, Roy A.: „Ritual und performative Sprache.“ In: Ritualtheorien. Ein einführendes Handbuch. Hg. v. Andréa Belliger u. David J. Krieger. Opladen, Wiesbaden 1998, 191-211.

Ratschow, Carl Heinz: „Gebet. Religionsgeschichtlich.“ In: TRE 12 (1993), 31-34.

Riesebrodt, Max: „Ethische und exemplarische Prophetie.“ In: Max Webers ,Religionssystematik'. Hg. v. Hans G. Kippenberg u. Max Riesebrodt. Tübingen 2001, 193-208.

Samarin, William J.: „Die Sprache der Religion und die Religionsforschung.“ In: Probleme der religiösen Sprache. Hg. v. Manfred Kaempfert. Darmstadt 1983, 273-292.

Seitz, Manfred: „Vaterunser. Kirchgeschichtlich und praktisch theologisch.“ In: TRE 34 (2002), 515-529.

Simmler, Franz: „Textsorten des religiösen und kirchlichen Bereichs.“ In: Textund Gesprächslinguistik. Ein internationales Handbuch zeitgenössischer Forschung. Textlinguistik Halbbd. 1. Hg. v. Klaus Brinker, Gerd Antos, Wolfgang Heinemann u. Sven Frederik Sager. Berlin, New York 2000, 676-690.

Simmler, Franz: „Luthers Evangelienübersetzung und die Entstehung neuer Makrostrukturen im Deutschen." In: Sprachwissenschaft 30 (2005a), 161-216.

Simmler, Franz: „Biblische Textsorten: ihre Merkmale und Traditionen von der Mitte des 15. bis zur Mitte des 16. Jahrhunderts.“ In: Daphnis 33 (2005b), 379-546.

Simmler, Franz: „Liturgische Textsorten und Textallianzen.“ In: Wissenschaften im Kontakt. Kooperationsfelder der Deutschen Sprachwissenschaft. Festschrift für Albrecht Greule. Hg. v. Sandra Reimann u. Katja Kessel. Tübingen 2007, 451-468. 
Soeffner, Hans-Georg: Die Ordnung der Rituale. Die Auslegung des Alltags - Der Alltag der Auslegung. Frankfurt/Main 1989.

Steger, Hugo: „Probleme der religiösen Sprache und des religiösen Sprechens.“ In: Sprechend nach Worten suchen. Probleme der philosophischen, dichterischen und religiösen Sprache der Gegenwart. Hg. v. Klaus Mönig. Freiburg/Breisgau 1984, 96-133.

Sundermeier, Theo: Was ist Religion? Religionswissenschaft im theologischen Kontext. Ein Studienbuch. Gütersloh 1999.

Turner, Viktor. W.: Das Ritual. Struktur und Antistruktur. Theorie und Gesellschaft 10. Frankfurt/Main 1989.

Turner, Viktor W.: „Liminalität und Communitas (1967).“ In: Ritualtheorien. Ein einführendes Handbuch. Hg. v. Andréa Belliger u. David J. Krieger. Opladen, Wiesbaden 1998, 251-262.

Tworuschka, Monika u. Udo Tworuschka: Islam Lexikon. Düsseldorf 2002.

Weber, Max: Wirtschaft und Gesellschaft. Grundriß der verstehenden Soziologie. Hg. v. Johannes Winckelmann. Tübingen $1976^{5}$.

Wengst, Klaus: „Glaubensbekenntnis(se). IV. Neues Testament.“ In: TRE 13 (1984), 392-399.

Wensinck, Arent Jan u. Johannes Hendrik Kramers (Hg.): Handwörterbuch des Islam. Leiden 1976.

Werlen, Iwar: Ritual und Sprache. Zum Verhältnis von Sprechen und Handeln in Ritualen. Tübingen 1984.

Werlen, Iwar: „Die ,Logik“ ritueller Kommunkation.“ In: Lili 60 (1987), 41-81. 
